Elmar Bollin | Klaus Huber | Dirk Mangold

\title{
Solare Wärme
}

für große Gebäude und Wohnsiedlungen

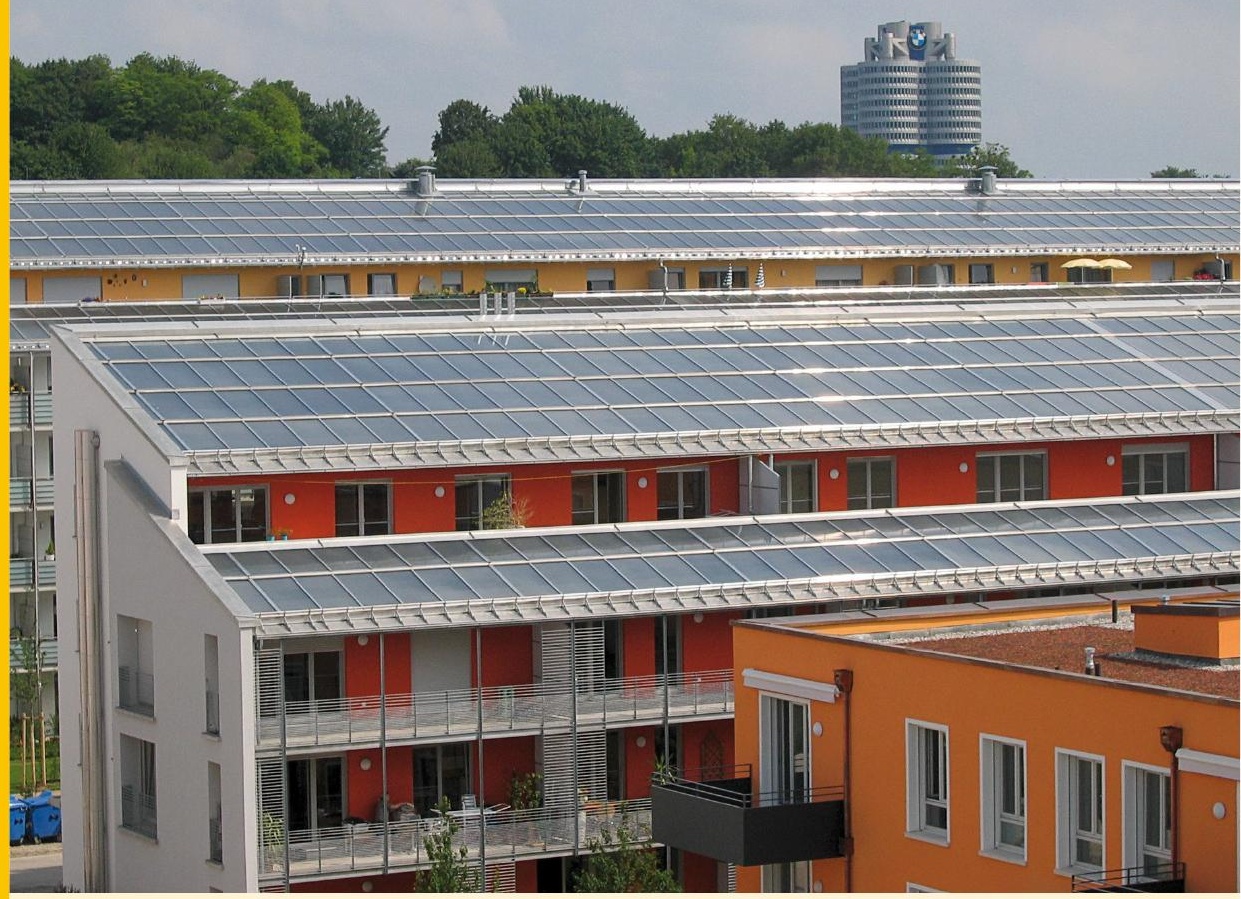

Fraunhofer IRB Verlag 
BINE-Fachbuch

Elmar Bollin, Klaus Huber, Dirk Mangold

Solare Wärme für große Gebäude und Wohnsiedlungen 
BINE-Fachbuch

\section{Solare Wärme für große Gebäude und Wohnsiedlungen}

Die Autoren:

Elmar Bollin

Klaus Huber

Dirk Mangold

Fachliche Beratung:

Reiner Croy 
BINE Informationsdienst berichtet über Themen der Energieforschung: Neue Materialien, Systeme und Komponenten, innovative Konzepte und Methoden. BINE-Leser werden so über Erfahrungen und Lerneffekte beim Einsatz neuer Technologien in der Praxis informiert. Denn erstklassige Informationen sind die Grundlage für richtungsweisende Entscheidungen, sei es bei der Planung energetisch optimierter Gebäude, der Effizienzsteigerung industrieller Prozesse oder bei der Integration erneuerbarer Energien in bestehende Systeme.

Weitere Informationen finden Sie unter: www.bine.info

Für weitere Fragen steht Ihnen zur Verfügung:

Uwe Friedrich (Redaktion)

BINE Informationsdienst, FIZ Karlsruhe, Büro Bonn

Kaiserstraße 185-197, 53113 Bonn

Tel. +49 228923 79-0, E-Mail: bine@fiz-karlsruhe.de, www.bine.info

Bibliografische Information der Deutschen Nationalbibliothek:

Die Deutsche Nationalbibliothek verzeichnet diese Publikation in der Deutschen Nationalbibliografie; detaillierte bibliografische Daten sind im Internet über www.dnb.de abrufbar.

ISBN (Print): 978-3-8167-8752-5 I ISBN (E-Book): 978-3-8167-8753-2

Layout: Dietmar Zimmermann I Umschlaggestaltung: Martin Kjer I Herstellung: Tim Oliver Pohl Satz: Mediendesign Späth GmbH, Birenbach I Druck: DZA Druckerei zu Altenburg GmbH, Altenburg

Alle Rechte vorbehalten.

Dieses Werk ist einschließlich aller seiner Teile urheberrechtlich geschützt. Jede Verwertung, die über die engen Grenzen des Urheberrechtsgesetzes hinausgeht, ist ohne schriftliche Zustimmung des Fraunhofer IRB Verlages unzulässig und strafbar. Dies gilt insbesondere für Vervielfältigungen, Übersetzungen, Mikroverfilmungen sowie die Speicherung in elektronischen Systemen.

Die Wiedergabe von Warenbezeichnungen und Handelsnamen in diesem Buch berechtigt nicht zu der Annahme, dass solche Bezeichnungen im Sinne der Warenzeichen- und Markenschutz-Gesetzgebung als frei zu betrachten wären und deshalb von jedermann benutzt werden dürften.

Die Inhalte dieses Werkes werden von Verlag, Herausgeber und Autoren nach bestem Wissen und Gewissen zusammengestellt. Eine rechtliche Gewähr für die Richtigkeit der einzelnen Angaben kann jedoch nicht übernommen werden. Es empfiehlt sich, gegebenenfalls für die eigenen Arbeiten die vollständigen Vorschriften oder Richtlinien in der jeweils gültigen Fassung hinzuzuziehen.

Titelbild: Neues Stadtquartier »Am Ackermannbogen«, München; () Landeshauptstadt München

Bilder Umschlagrückseite (von links):

Elmar Bollin; Dirk Mangold; Marstal Fjernwarme, Marstal (Dänemark)

(c) by FIZ Karlsruhe

Verlag und Vertrieb:

Fraunhofer IRB Verlag

Fraunhofer-Informationszentrum Raum und Bau IRB

Nobelstraße 12, 70569 Stuttgart

Telefon $+49711970-2500$

Telefax $+49711970-2508$

irb@irb.fraunhofer.de

www.baufachinformation.de

Hinweis zu den Abbildungen: Soweit nachfolgend keine anderen Quellen genannt werden, stammen die Abbildungen von den Autoren. 


\section{Vorwort}

Der solaren Wärme kommt im künftigen Wärmeenergiemix eine große Bedeutung zu. Die Anzahl der Anlagen muss deutlich ausgebaut, der durchschnittliche Solaranteil an der Wärmebedarfsdeckung eines Gebäudes oder eines Quartiers weiter erhöht und bislang brachliegende Anwendungsfelder müssen systematisch erschlossen werden. Die Breitenförderung konzentrierte sich bislang vor allem auf Solarwärmeanlagen in Ein- und Zweifamilienhäusern; ein Großteil der Solarkollektoren wird hier eingesetzt. Das große Potenzial im Mietwohnungsbereich, in Hotels, Krankenhäusern, Altenheimen, Gewerbebetrieben, aber auch in Wohnsiedlungen und Gewerbegebieten ist dagegen kaum erschlossen. Und dies trotz beachtlicher Fortschritte in der Technologieentwicklung.

Denn die für große solarthermische Anlagen zur solar unterstützten Trinkwassererwärmung entwickelte und in über 50 Pilot- und Demonstrationsanlagen erprobte Systemtechnik ist inzwischen marktreif. Mit dem Forschungsprogramm "Solarthermie-2000 « und dem Nachfolge-Förderprogramm »Solarthermie2000plus « unterstützt die Bundesregierung seit 1993 die Entwicklung dieser Technik und den Bau von Demonstrationsanlagen. Daneben widmete sich die Forschung dem Anlagen-Monitoring, der Funktions- und Ertragskontrolle sowie neuen Lösungen in der Solarthermietechnik. Die 2007 erstellte GroSol-Studie des Bundesverbands Solarwirtschaft hatte den Abbau rechtlicher und organisatorischer Markteintrittsbarrieren zum Ziel.

Die solar unterstützte Nahwärme bedeutet den Einstieg in eine dauerhaft zukunftsfähige Wärmeversorgung größerer Liegenschaften oder ganzer Siedlungen. In vielen Szenarien zur künftigen Integration erneuerbarer Energien spielt sie im Wärmemarkt mittel- bis langfristig eine bedeutende Rolle. Ihre technische Umsetzung ist für viele Beteiligte jedoch oft noch Neuland und verursacht moderate Mehrkosten im Vergleich zu konventionellen Nahwärmesystemen.

Die ersten Pilotanlagen solar unterstützter Nahwärmenetze mit saisonalem Wärmespeicher gingen 1996 in Betrieb und haben inzwischen den Nachweis der Funktionsfähigkeit bzw. der Umsetzung im Siedlungsmaßstab erfolgreich erbracht. Den Technologiefortschritt der letzten 15 Jahre für die Behälter-, Erdbecken und Erdsonden-Wärmespeicher fasst die vorliegende Publikation kenntnisreich zusammen und zeigt die derzeitigen Entwicklungslinien auf. Zukunftsperspektiven ergeben sich für große Kollektorflächen zur direkten Einbindung in Fernwärmenetze, für solar unterstützte Nahwärmenetze als Insellösungen und insbesondere für großvolumige Wärmespeicher, die zukünftig nicht nur Solarwärme saisonal speichern, sondern multifunktional genutzt werden, um neben der Wärmeversorgung durch Solarthermie auch die Stromversorgung zu optimieren und abzusichern. Solche "Smart-District-Heating «Systeme stehen jedoch noch am Anfang.

Vor diesem Hintergrund bietet das BINE-Fachbuch einen fundierten Überblick über Anlagentechnik, Systemauslegung, Wirtschaftlichkeit und ausgewählte Pilotanwendungen für große Gebäude und Wohnsiedlungen. In kompakter Form arbeitet es die Erfahrungen der Förderprogramme "Solarthermie-2000 « und "Solarthermie2000plus « auf und widmet sich aktuellen Entwicklungen wie Zukunftsperspektiven.

Autoren des ersten Teils, der sich mit "Solaranlagen in großen Gebäuden « befasst, sind Prof. Elmar Bollin und Klaus Huber vom Lehrstuhl für Gebäudeautomation, Haustechnik und Solartechnik an der FH Offenburg. Der zweite Teil »Solar unterstützte Nahwärme und saisonale 
Wärmespeicher « stammt von Dirk Mangold, Institutsleiter von Solites. Ihm oblag die wissenschaftliche Programmleitung für solare Nahwärme und saisonale Wärmespeicher des bundesweiten Förderprogramms Solarthermie2000plus.

FIZ Karlsruhe

BINE Informationsdienst 


\section{Inhaltsverzeichnis}

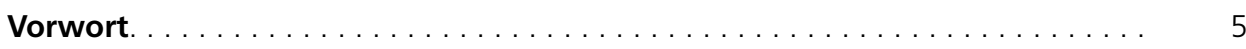

Teil 1 | Solaranlagen in großen Gebäuden und im Geschosswohnungsbau . 11

1 Grundlagen solarthermischer Energienutzung in großen Gebäuden und $\mathrm{im}$ Geschosswohnungsbau. . . . . . . . . . . . . . . . . 13

1.1 Charakteristik großer Solarthermie-Anlagen. . . . . . . . . . . . . . . . . . . 14

1.2 Unterschiede zu anderen regenerativen Wärmeversorgungssystemen . . . . . . 15

1.3 Solarthermie-Anlagen in der Energieeinsparverordnung (EnEV) . . . . . . . . 16

1.4 Ergebnisse der Marktstudie zu großen Solarwärmeanlagen (GroSol) . . . . . . . 18

2 Systemtechnik großer Solarthermie-Anlagen . . . . . . . . . . . . . 21

2.1 Solare Vorwärmesysteme. . . . . . . . . . . . . . . . . . . . . 21

2.2 Besonderheiten beim Betrieb solarthermischer Systeme . . . . . . . . . . . 23

2.3 Nützliche Werkzeuge bei Planung und Betrieb solarthermischer Großanlagen . 26

$3 \quad$ Anlagentechnik. . . . . . . . . . . . . $\ldots \ldots \ldots \ldots \ldots$

$3.1 \quad$ Kollektorbauarten und Kollektorfelder. . . . . . . . . . . . . . . . 33

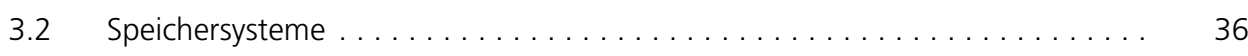

3.3 Wärmeübertrager . . . . . . . . . . . . . . . . . . . . . . . 39

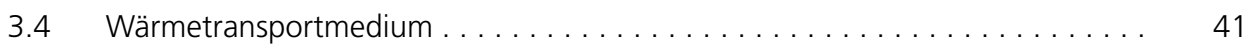

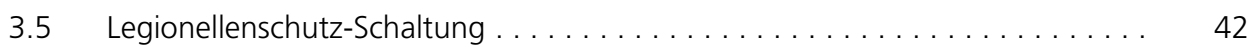

3.6 Sicherheit solarthermischer Anlagen . . . . . . . . . . . . . . . . . 43

4 Solarthermische Anwendungen in Gebäuden . . . . . . . . . . . . . 47

4.1 Trinkwassererwärmungssysteme . . . . . . . . . . . . . . . . 47

4.2 Empfehlungen für die Auslegung solarer Trinkwarmwassersysteme . . . . . . . . 53

4.3 Anlagen zur kombinierten Trinkwassererwärmung und Gebäudeheizung

$5 \quad$ Wirtschaftlichkeit solarthermischer Großanlagen . . . . . . . . . . 59

5.1 Methodischer Ansatz zur Untersuchung der Wirtschaftlichkeit . . . . . . . . 59

$5.2 \quad$ Kosten und Erträge . . . . . . . . . . . . . . . . . . . . . 60

5.3 Solare Nutzwärmekosten im Vergleich. . . . . . . . . . . . . . . . . . . 62

$6 \quad$ Ausgewählte Anlagenbeispiele. . . . . . . . . . . . . . 65

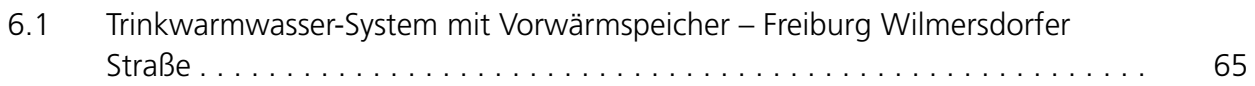

6.2 Frischwassersystem: Trinkwassererwärmung ohne Vorwärmspeicher in 
6.3 Anlagen zur kombinierten Trinkwassererwärmung und Gebäudeheizung (solar unterstützte Nahwärmeversorgung), Holzgerlingen . . . . . . . . . 73

6.4 Vergleichende Betrachtung großer solarthermischer Anlagen. . . . . . . . . . 77

Teil 2 | Solar unterstützte Nahwärme und saisonale Wärmespeicher. . . . . . 83

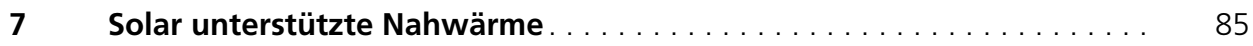

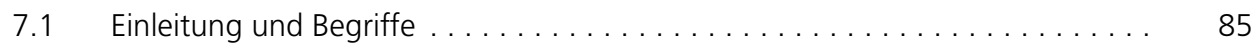

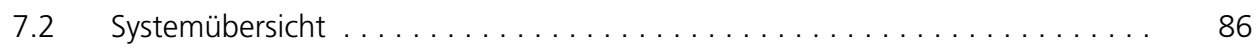

$8 \quad$ Komponenten solarer Nahwärmesysteme . . . . . . . . . . . . . . . 91

$8.1 \quad$ Kollektorfeld und Solarkreis . . . . . . . . . . . . . . . . . . . . . . . . . . . 91

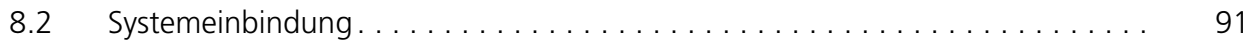

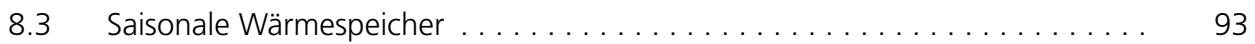

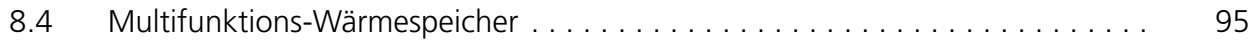

$9 \quad$ Ausgewählte Pilotanlagen. . . . . . . . . . . . . . . . . . . . 97

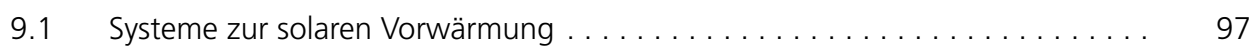

9.2 Solar unterstützte Nahwärme mit Kurzzeit-Wärmespeicher in Neckarsulm . . . . 98

9.3 Übersicht der Pilotanlagen mit saisonalem Wärmespeicher. . . . . . . . . . . . . . 99

9.4 Solar unterstützte Nahwärme mit saisonalem Wärmespeicher

$\begin{aligned} 9.5 & \text { Solar unterstützte Nahwärme mit saisonalem Wärmespeicher } \\ & \text { »Hirtenwiesen } \| \ll \text { in Crailsheim } \ldots \ldots \ldots \ldots \ldots \ldots \ldots \ldots \ldots \ldots \ldots \ldots \ldots \ldots \ldots \ldots\end{aligned}$

$10 \quad$ Planungshinweise. . . . . . . . . . . . . . . . . . . . . 121

10.1 Entwicklungsstand der Teilsysteme. . . . . . . . . . . . . . . . . . 121

10.2 Große Kollektorfelder für solar unterstützte Nahwärmesysteme . . . . . . . . . . 123

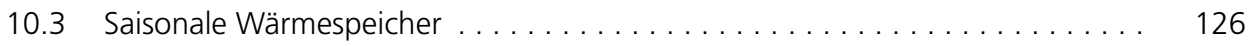

10.4 Systemsimulation und -dimensionierung . . . . . . . . . . . . . . . 129

10.5 Rechtliche Aspekte der Realisierung von Pilotanlagen . . . . . . . . . . . . 130

11 Wirtschaftlichkeit und Perspektiven solarer Nahwärmesysteme . . . . . 133

11.1 Wirtschaftlichkeit und Kosten . . . . . . . . . . . . . . . . . . 133

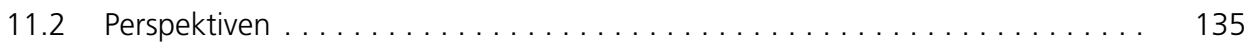

Serviceteil . . . . . . . . . . . . . . . . . . . . . . . . 139

12 Zitierte Literatur und Abbildungsverzeichnis . . . . . . . . . . . . . . . 141

12.1 Zitierte Literatur. . . . . . . . . . . . . . . . . . . . . . . 141

12.2 Abbildungsverzeichnis . . . . . . . . . . . . . . . . . . . . 142 
13 Forschungsvorhaben der Bundesregierung

13.1 Forschungsvorhaben im Rahmen des Förderprogrammes Solarthermie-2000, TP 2 »Große Solaranlagen« . . . . . . . . . . . . . . . . . . . . . . . . . . . . . . . 145

13.2 Forschungsvorhaben im Rahmen des Förderprogrammes Solarthermie-2000, TP 3 »Solar unterstützte Nahwärme« und Solarthermie2000plus . . . . . . . . . . 150

14 Weiterführende Literatur. . . . . . . . . . . . . . . . . . . . 155

14.1 Literatur . . . . . . . . . . . . . . . . . . . . . . . . . . . . . . 155

14.2 Sonstige, von den Autoren empfohlene Literatur und Internet-Links . . . . . . . 156

14.3 BINE Informationsdienst. . . . . . . . . . . . . . . . . . . . . . . . . . . 157

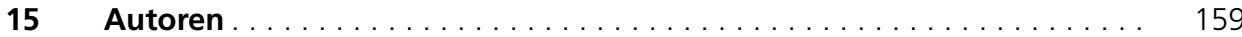


